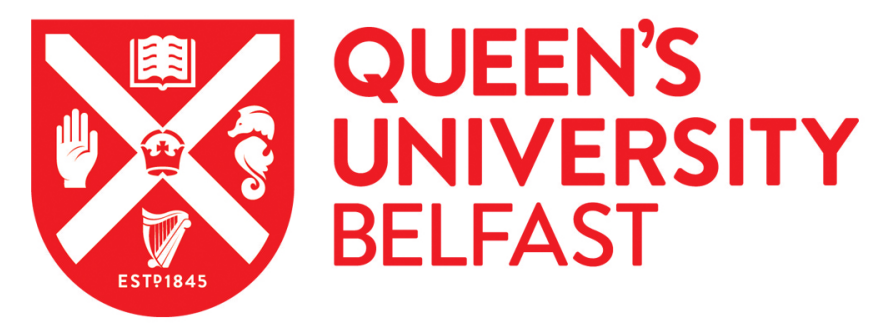

\title{
Event Detection in Molecular Communication Networks with Anomalous Diffusion
}

Mai, T. C., Egan, M., Duong, Q., \& Di Renzo, M. (2017). Event Detection in Molecular Communication Networks with Anomalous Diffusion. IEEE Communications Letters, 21(6), 1249-1252.

https://doi.org/10.1109/LCOMM.2017.2669315

\section{Published in:}

IEEE Communications Letters

\section{Document Version:}

Peer reviewed version

Queen's University Belfast - Research Portal:

Link to publication record in Queen's University Belfast Research Portal

\section{Publisher rights}

Copyright 2017 IEEE.

This work is made available online in accordance with the publisher's policies. Please refer to any applicable terms of use of the publisher.

\section{General rights}

Copyright for the publications made accessible via the Queen's University Belfast Research Portal is retained by the author(s) and / or other copyright owners and it is a condition of accessing these publications that users recognise and abide by the legal requirements associated with these rights.

Take down policy

The Research Portal is Queen's institutional repository that provides access to Queen's research output. Every effort has been made to ensure that content in the Research Portal does not infringe any person's rights, or applicable UK laws. If you discover content in the Research Portal that you believe breaches copyright or violates any law, please contact openaccess@qub.ac.uk. 


\title{
Event Detection in Molecular Communication Networks with Anomalous Diffusion
}

\author{
Trang C. Mai, Malcolm Egan, Trung Q. Duong, and Marco Di Renzo
}

\begin{abstract}
A key problem in nanomachine networks is how information from sensors is to be transmitted to a fusion center. In this paper, we propose a molecular communicationbased event detection network. In particular, we develop a detection framework that can cope with scenarios where the molecules propagate according to anomalous diffusion instead of the conventional Brownian motion. We propose an algorithm for optimizing the network throughput by exploiting tools from reinforcement learning. Our algorithms are evaluated with the aid of numerical simulations, which demonstrate the tradeoffs between performance and complexity.
\end{abstract}

\section{INTRODUCTION}

Networks consisting of a large number of nanomachines, which are able to sense, communicate, and actuate at the nanoscale, have been proposed for applications ranging from intrabody health monitoring to pollution control [1]. A key component of these networks is the detection of events such as the presence of undesirable chemicals in the atmosphere or the malfunction of cells in biological systems. In a network of distributed nanomachines, this information must then be sent to a fusion nanomachine $(\mathrm{FN})$, which can take action to mitigate the effect of the event.

Molecular communication forms one approach to support communication among sensing nanomachines (SNs) and a FN, where unlike conventional electromagnetic-based communication, each SN encodes information in the release time, number, or type of molecules emitted by each SN [1]-[3]. Molecular communication raises new challenges, due to the low energy and limited computational resources available at each nanomachine. Moreover, the achievable throughput of molecular communication systems is limited by the noise introduced from the random diffusion time of each molecule.

In existing works, the diffusion is typically modeled according to Brownian motion. In this case, it is possible to derive closed-form expressions for the first passage time distribution and the impulse response for reasonable boundary conditions. Under the assumption of Brownian motion, capacity characterizations [4], [5], practical receiver designs [6], [7] and intersymbol interference mitigation strategies [8] have been proposed.

This work was supported in part by the U.K. Royal Academy of Engineering Research Fellowship under Grant RF1415 \14\22.

T. C. Mai and T. Q. Duong are with the Institute of Electronics, Communications and Information Technology in Queen's University Belfast, Belfast, U.K. (email: \{tmai01, trung.q.duong\} @qub.ac.uk)

M. Egan is with Univ Lyon, INSA Lyon, INRIA, CITI, France (email: malcolm.egan@gmail.com )

M. Di Renzo is with the Laboratory of Signals and Systems, Central SuplecUniversity Paris-Sud XI, 91400 Orsay, France (email: marco.direnzo@1ss.supelec.fr)
However, Brownian motion cannot capture anomalous diffusion, where the growth of the mean square displacement is much faster or slower as it usually occurs in scenarios with turbulence [9]. A key class of models for anomalous diffusion are the Lévy walks, which introduce challenges for the design of reliable molecular communication systems, as the first passage time and the impulse response are not generally available in closed-form. Moreover, the diffusion process cannot be encapsulated in a small number of parameters in Lévy walk models, as is the case in Brownian motion. These challenges are exacerbated in the context of event detection, where the large number of $\mathrm{SNs}$ lead to a corresponding number of diffusion channels, each with a different distance and fluid properties between the transmitting and receiving nanomachines. In particular, existing molecular communication techniques for Brownian motion and a single transmitting SN cannot be directly applied.

In this paper, we propose a molecular communication system for event detection which can cope with anomalous diffusion. Our system consists of a event detector and a training phase to optimize the time slot duration and tune the detection rule. In particular, we design the training phase to estimate the parameters required for reliable detection based on one bit messages from each SN. To design the training phase, we introduce a reinforcement learning-based algorithm for optimizing the time slot duration. In the proposed algorithm, the duration of each time slot is chosen in order to maximize the throughput and is applicable to any diffusion process with stationary increments and an independent first passage time for each link between a $\mathrm{SN}$ and the $\mathrm{FN}$. In particular, it can be applied to the Lévy walks. To evaluate the performance of the system, we derive a tractable expression for the probability of error and numerically investigate tradeoffs between performance and complexity.

\section{SYSTEM MODEL}

We consider a synchronized and time-slotted nanoscale network, where $N_{S}$ SNs monitor the state of a system. The duration of each time slot is $\mathcal{T}_{S}$. When a SN observes an event, it emits a molecule at the beginning of the next transmission slot. The intended receiver of the message is the FN, which processes the messages from the SNs. For example, the FN might be the head of a cluster of SNs which sends the detection of an event to an actuator nanomachine.

Events occur with probability $p_{E}$ and we assume that each SN detects an event with probability $p_{D}$. The molecule transmitted by each $\mathrm{SN}$ diffuses to the FN according to a 
Lévy walk [10]. We assume that the FN perfectly absorbs any detected molecule. As we will see in further text, our system design is applicable to any network of SNs where the diffusion process is stationary and the first passage time of each SN-FN link is independent. In particular, the proposed design applies both to Brownian motion and to Lévy walks under a wide range of boundary conditions. As Lévy walks form a very general class of diffusion processes, to make our description concrete, we assume that the $\mathrm{FN}$ is an absorbing boundary, the Lévy walk is one-dimensional, and the distance that a molecule travels within time $t$ is given by

$$
d_{t}=\sum_{j=1}^{n_{t}} v D_{j},
$$

where $n_{t}$ is the number of jumps within time $t, D_{j} \in \mathbb{R}$ is the duration and direction of the $j$-th jump, and $v$ is the fixed velocity. We assume that $D_{j}$ is an $\alpha$-stable random variable with exponent $1<\alpha<2$, scale parameter $\sigma \in \mathbb{R}_{>0}$, skewness $\beta=0$, and shift $\delta=0$.

Molecules that are emitted in earlier time slots and that are not absorbed by the FN persist. As a consequence, there is inter-symbol interference and the number of molecules observed by the $\mathrm{FN}$ in time slot $j$ is given by $\sum_{k=1}^{j} Y_{k}^{j}$, where $Y_{k}^{j}=\sum_{i=1}^{N_{S}} Y_{k, i}^{j}$ is the number of molecules that are transmitted in time slot $k$ and that arrive in time slot $j$. In particular, the random variable $Y_{k, i}^{j}$ is Bernoulli distributed with success occurring when an event occurs and the molecule sent from the $i$-th $\mathrm{SN}$ in slot $k$ arrives at the $\mathrm{FN}$ in slot $j$. Let $F(t)$ be probability that a molecule transmitted at time 0 is absorbed before time $t$. Then the success probability is equal to

$$
P_{j-k, i}=F\left((j+1-k) \mathcal{T}_{S}\right)-F\left((j-k) \mathcal{T}_{S}\right),
$$

where $P_{j-k, i}$ is the probability that $(j-k)$ time slots for the molecule from SN $i$ to diffuse to the $\mathrm{FN}$ are needed. Based on these assumptions, the inter-symbol interference is given by $\sum_{k=1}^{j-1} Y_{k}^{j}$ and the desired signal is $Y_{j}^{j}$.

\section{Detection Rule AND ERROR Probability}

To determine whether or not an event has occurred, the FN solves a hypothesis-testing problem based on the number of observed molecules. Let $H_{1}$ be the hypothesis that an event occur and $H_{0}$ be the hypothesis that an event did not occur. The number of molecules that arrive in time slot $j$ under each hypothesis is given by

$$
\begin{aligned}
& H_{0}: R_{H_{0}}=\sum_{k=1}^{j-1} Y_{k}^{j} \\
& H_{1}: R_{H_{1}}=Y_{j}^{j}+\sum_{k=1}^{j-1} Y_{k}^{j},
\end{aligned}
$$

For an observation of $r$ molecules, the detection rule is given by the likelihood ratio test [11]

$$
\frac{\operatorname{Pr}\left(r \mid H_{1}\right)}{\operatorname{Pr}\left(r \mid H_{0}\right)} \sum_{H_{0}}^{H_{1}} \eta,
$$

where $\eta=\left(1-p_{E}\right) / p_{E}$ and the costs are $C_{00}=C_{11}=0$ and $C_{01}=C_{10}=1$.

As observed in [6], $Y_{k, i}^{j}$, which is the event that the molecule emitted in time slot $k$ by SN $i$ that arrive in time slot $j$, is a Bernoulli random variable with success probability $P_{j-k, i}$. It then follows that $Y_{k}^{j}=\sum_{i=1}^{N_{S}} Y_{k, i}^{j}$ is binomial distributed. For finite $j, R_{H_{0}}$ and $R_{H_{1}}$, which are sums of independent binomial distributions, are Poisson-binomial distributed. Moreover, for systems operating for long periods of time corresponding to $j \rightarrow \infty$, it follows from the law of rare events [12], [13] that $R_{H_{0}}$ and $R_{H_{1}}$ converge in distribution to a Poisson random variable. The large $j$ approximation also forms a worst case scenario for short system run times.

To derive the parameter of the Poisson random variable, we note that an event occurs and is detected by $\mathrm{SN} i$ with probability $p_{E} p_{D}$. Moreover, $\sum_{k=0}^{j} P_{j-k, i}=1$ when $j \rightarrow \infty$. This means that the inter-symbol interference due to $\mathrm{SN} i$ is a Poisson random variable with expected value $p_{E} p_{D}\left(1-P_{0, i}\right)$, where $P_{0, i}$ is the probability that a single time slot is needed for a molecule emitted by $\mathrm{SN} i$ to reach the FN. Since the sum of Poisson random variables is Poisson, it follows that

$$
\begin{aligned}
& R_{H_{0}} \sim \text { Poisson }\left(p_{E} p_{D} \sum_{i=1}^{N_{S}}\left(1-P_{0, i}\right)\right) \\
& R_{H_{1}} \sim \text { Poisson }\left(\sum_{i=1}^{N_{S}} P_{0, i}+p_{E} p_{D} \sum_{i=1}^{N_{S}}\left(1-P_{0, i}\right)\right) .
\end{aligned}
$$

We are now in the position to derive a decision rule. Let

$$
\begin{aligned}
& \lambda_{0}=p_{E} p_{D} \sum_{i=1}^{N_{S}}\left(1-P_{0, i}\right) \\
& \lambda_{1}=\sum_{i=1}^{N_{S}} P_{0, i}+p_{E} p_{D} \sum_{i=1}^{N_{S}}\left(1-P_{0, i}\right) .
\end{aligned}
$$

By using the probability density function of the Poisson distribution in (4), we obtain the decision rule

$$
r \underset{H_{0}}{\stackrel{H_{1}}{\gtrless}} \Theta
$$

where

$$
\Theta=\frac{\log \eta+\lambda_{1}-\lambda_{0}}{\log \left(\lambda_{1}\right)-\log \left(\lambda_{0}\right)} .
$$

Under the Poisson approximation, the average probability of detection error is given by

$$
P_{e}=p_{E} F\left(\lambda_{1} ;\lfloor\Theta\rfloor\right)+\left(1-p_{E}\right)\left(1-F\left(\lambda_{0} ;\lceil\Theta\rceil\right)\right),
$$

where $F(\lambda ; x)$ is the CDF of the Poisson distribution. Since Poisson-binomial random variables converges in distribution to a Poisson random variable under the conditions [12] satisfied by our model, it follows that the probability of error converges to $(9)$.

A key observation is that the decision rule and hence the error probability are completely determined by the parameter

$$
A=\sum_{i=1}^{N_{S}} P_{0, i} .
$$


We remark that if Brownian motion is considered, it is possible to obtain an analytical expression for $P_{0, i}$, under appropriate boundary conditions, and perfect knowledge of the fluid diffusion coefficient as well as the distances between each SN and the FN. However, in the case of Lévy walks, a closed-form expression for the first passage time is not available. Moreover, due to the large number of SNs, it is unlikely that the FN will have access to the necessary information on the fluid properties and locations of all the SNs throughout the network. In the next section, we explore the effect of the training phase on the estimate of $A$ and the decision rule.

\section{Training Phase Design}

Due to the unknown diffusion process, the training phase plays a crucial role in obtaining the parameters that are required for the decision rule. In this section, we design the training phase to ensure that the probability of error lies within a known confidence interval. We then exploit the confidence interval to develop an online algorithm inspired by reinforcement learning [14], to optimize the time slot duration. By exploiting reinforcement learning, our approach optimizes the time slot duration by exploiting observations from sensor signals without having a complete characterization of the channel. As the first passage time and the impulse response in our model are not available in closed-form, such a learningbased approach is highly desirable.

To develop an online algorithm, we assume that after each time slot in the training phase, the network can remove the transmitted molecules from the system. This can be achieved by the introduction of reactants, such as the enzyme-based approach in [8], which reduce the lifetime of the information molecules, only necessary during the training phase to reduce the energy consumption at the $\mathrm{FN}$ while achieving accurate parameter estimation.

From (10), it follows that the probability of error is completely determined by the parameter $A$ in (10). As a consequence, the key step in the training phase is to estimate $A$. A natural estimator is

$$
\hat{A}=\sum_{i=1}^{N_{S}} \frac{1}{n} \sum_{j=1}^{n} \mathbf{1}_{\left\{Y_{i, j}=1\right\}},
$$

where $Y_{i, j} \in\{0,1\}$ is the number of molecules that are received within a single time slot from the $i$-th $\mathrm{SN}$ in the $j$-th training slot. Let us define

$$
\hat{P}_{0, i}=\frac{1}{n} \sum_{j=1}^{n} \mathbf{1}_{\left\{Y_{i, j}=1\right\}},
$$

which is an empirical distribution function. Thus, it follows that the estimator $\hat{A}$ is consistent [15]. Moreover, we observe that

$$
\begin{aligned}
\mathbb{P}\left(|A-\hat{A}|>N_{S} \epsilon\right) & \leq \mathbb{P}\left(\sum_{i=1}^{N_{S}}\left|P_{0, i}-\hat{P}_{0, i}\right|>N_{S} \epsilon\right) \\
& \leq 1-\prod_{i=1}^{N_{S}}\left(1-\mathbb{P}\left(\left|P_{0, i}-\hat{P}_{0, i}\right|>\epsilon\right)\right)
\end{aligned}
$$

Based on these considerations, we can apply the DvoretzkyKiefer-Wolfowitz inequality [15], which yields

$$
\begin{aligned}
\mathbb{P}\left(|A-\hat{A}|>N_{S} \epsilon\right) & \leq 1-\prod_{i=1}^{N_{S}}\left(1-2 e^{-2 n \epsilon^{2}}\right) \\
& =1-\left(1-2 e^{-2 n \epsilon^{2}}\right)^{N_{S}} .
\end{aligned}
$$

Let the time slot duration be $\mathcal{T}_{S} \in\left\{\mathcal{T}_{1}, \ldots, \mathcal{T}_{K}\right\}$. The bound in (14) provides a means of obtaining the confidence intervals for the probability of error and the throughput, which is defined as

$$
R_{l}=\frac{1}{\mathcal{T}_{l}}\left(1-P_{e, l}\right),
$$

for the $l$-th time slot duration, where $P_{e, l}$ is the probability of error corresponding to a time slot duration $\mathcal{T}_{l}$. In particular, given an estimate $\hat{A}_{l}$ it follows that the probability $A_{l} \in\left[\hat{A}_{l}-\right.$ $\left.N_{S} \epsilon, \hat{A}_{l}+N_{S} \epsilon\right]$ is bounded by (14). Note that $P_{e, l}$ in (9) is a monotonically decreasing function of $A$, which is due to the fact that the number of molecules that arrive in a single time slot is increasing as $A$ increases.

Using the fact that $P_{e, l}$ is a monotonically decreasing function of $A$ and (14), the confidence interval bound on $R_{j}$

$$
\frac{1-P_{e, l}\left(\hat{A}_{l}-N_{S} \epsilon(\phi)\right)}{\mathcal{T}_{l}} \leq R_{l} \leq \frac{1-P_{e, l}\left(\hat{A}_{l}+N_{S} \epsilon(\phi)\right)}{\mathcal{T}_{l}},
$$

holds with probability greater than $1-\phi$, where

$$
\epsilon(\phi)=\sqrt{\frac{1}{2 n} \log \left(\frac{2}{1-\sqrt[N]{S}-\phi}\right)} .
$$

The confidence interval in (16) bounds the error probability and throughput with $n$ trials, which is useful for providing guarantees on the throughput given a limited training phase. The confidence interval also provides a means for optimizing the time slot duration. Since it is undesirable to obtain tight confidence intervals for every time slot duration, we can use the confidence interval as the basis for an online optimization algorithm. The algorithm can provide guarantees for the probability that the selected time slot duration is suboptimal.

We now present our online algorithm to minimize the probability that a suboptimal time slot duration is selected, as opposed to the time-consuming approach of estimating the throughput for each time slot duration separately. Let $0<\phi<1$ be the confidence level in (16) and the stopping criterion is $\delta>0$. Suppose that the training phase is in the $k$-th time slot, then:

1) If $1 \leq k \leq K$, set $j=k$ and select time slot duration $\mathcal{T}_{k}$. Using (11), compute the estimate $\hat{A}_{k}$ and the confidence interval via (16).

2) If $k>K$, select the time slot duration $j$ such that

$$
\frac{1}{\mathcal{T}_{j}}\left(1-P_{e, j}\left(\hat{A}_{j}+N_{S} \epsilon(\phi)\right)\right)
$$

is maximized.

The training phase is ended if for the selected time slot $\mathcal{T}_{j}$, the stopping criterion

$$
\frac{1}{\mathcal{T}_{j}}\left(P_{e, j}\left(\hat{A}_{j}-N_{S} \epsilon(\phi)\right)-P_{e, j}\left(\hat{A}_{j}+N_{S} \epsilon(\phi)\right)\right)<\delta
$$


is satisfied and the intersection of the confidence intervals defined by (16) is empty. Otherwise, the algorithm continues with $k \leftarrow k+1$.

Note that the algorithm is based on the confidence interval estimate in (16), Thus, it follows that the probability that the time slot duration $\mathcal{T}_{j}$ selected by the algorithm is suboptimal based on (9) is upper bounded by $\phi K$.

We remark that the estimation procedure is based on the estimation of the cumulative distribution function for the first passage time. Hence, our algorithm can be applied to systems with any diffusion process as long as the increments are independent and the first passage times for each SN-FN link are each independent.

\section{NumericAl RESUlts}

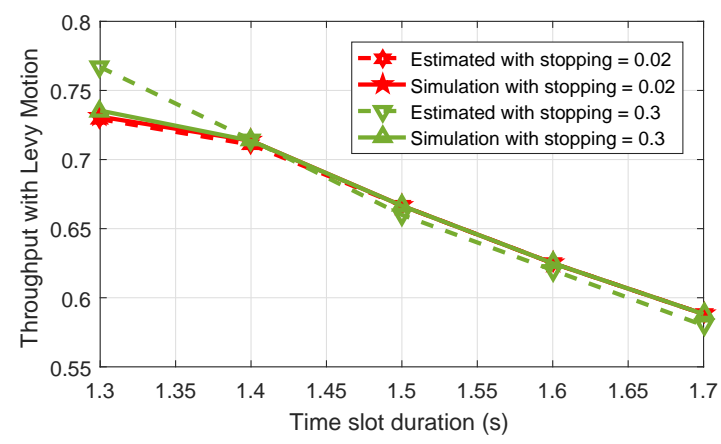

Fig. 1. Throughput with different stopping criteria.

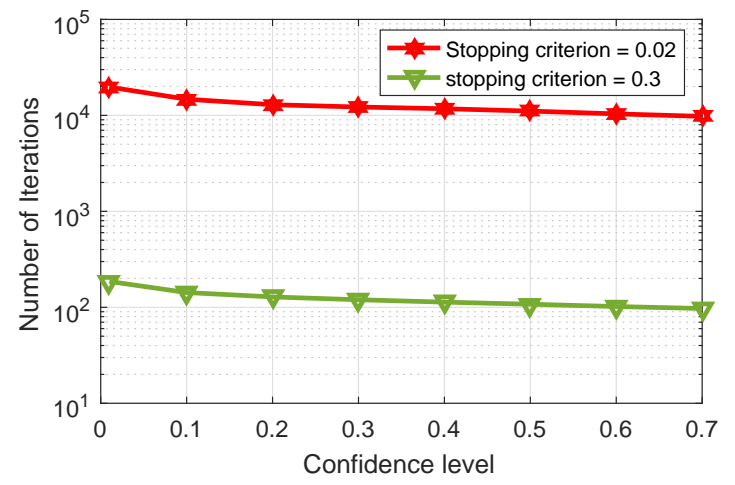

Fig. 2. Number of iterations with different stopping criteria.

In this section, we show the tradeoffs between performance and complexity of our algorithm by comparing its robustness with time duration spent on the training phase. The system consists of a single FN and $N_{S}=100$ transmitting SNs located at equal distance $d=10^{-2} \mathrm{~m}$ from the FN. To illustrate the behavior of our algorithm in the presence of anomalous diffusion, we consider a fluid medium with a drift velocity $v=0.01 \mathrm{~m} / \mathrm{s}, p_{E}=0.5, p_{D}=1$, stability exponent $\alpha=1.2$ and scale parameter $\sigma=0.01$ for each SN-FN link. We assume that the network can select a time slot duration in the set $\{1.3,1.4,1.5,1.6,1.7\}$.

Fig. 1 compares the throughput for each time slot duration obtained directly from Monte Carlo simulations and from the estimated parameter $\hat{A}$ using our algorithm for each time slot duration separately with a confidence level $\phi=0.01$. We observe that the estimate from our algorithm is in good agreement with the throughput obtained from Monte Carlo simulations. This implies that our algorithm can obtain the optimal throughput with probability dependent on the stopping time.

Fig. 2 plots the complexity in terms of the number of iterations for the training period as the confidence level varies, which determines the probability the selected throughput is suboptimal. We observe that the stopping criterion has a large influence on the number of iterations. Despite this, we can still obtain an accurate estimate of the throughput in our setting with a small number of iterations as observed in Fig. 1.

\section{CONCLUSION}

We have proposed an algorithm to optimize molecular communication in nanomachine sensor networks in the presence of a medium with anomalous diffusion. A key feature of our algorithm is that it does not rely on detailed statistical knowledge of the underlying diffusion processes and that it provides a guarantee on the probability that the throughput is optimal. Simulation results suggest that good performance can be obtained with very short training periods.

\section{REFERENCES}

[1] T. Nakano, M. Moore, F. Wei, A. Vasilaks, and J. Shuai, "Molecular communication and networking: opportunities and challenges," IEEE Trans. on Nanobioscience, vol. 11, no. 2, pp. 135-148, 2012.

[2] I. Akyildiz, F. Fekri, R. Sivakumar, C. Forest, and B. Hammer, "MoNaCo: fundamentals of molecular nano-communication networks," IEEE Wireless Commun., vol. 19, no. 5, pp. 12-18, 2012.

[3] N. Farsad, H. B. Yilmaz, A. Eckford, C. B. Chae, and W. Guo, "A comprehensive survey of recent advancements in molecular communication," IEEE Commun. Surveys Tutorials, vol. 18, no. 3, pp. 1887-1919, 2016.

[4] K. V. Srinivas, A. W. Eckford, and R. S. Adve, "Molecular communication in fluid media: The additive inverse gaussian noise channel," IEEE Trans. Inf. Theory, vol. 58, no. 7, pp. 4678-4692, July 2012.

[5] M. Egan et al., "Variance-constrained capacity of the molecular timing channel with synchronization error," in IEEE GLOBECOM, Austin, TX, Dec. 2014, pp. 1473-1478.

[6] H.-J. Chiu, L. S. Meng, P. C. Yeh, and C. H. Lee, "Near-optimal low complexity receiver design for diffusion-based molecular communication,” in IEEE GLOBECOM, Atlanta GA, Dec. 2013, pp. 3372-3377.

[7] B. Li et al., "Local convexity inspired low-complexity noncoherent signal detector for nanoscale molecular communications," IEEE Trans. Commun., vol. 64, no. 5, pp. 2079-2091, May 2016.

[8] A. Noel, C. Cheung, and R. Schober, "Improving receiver performance of diffusive molecular communication with enzymes," IEEE Trans. on NanoBioscience, vol. 13, no. 1, pp. 31-43, 2014.

[9] J. Klafter, M. Shleshinger, and G. Zumofen, "Beyond Brownian motion," Physics Today, vol. 49, no. 2, pp. 33-39, 1996.

[10] V. Zaburdaev, S. Denisov, and J. Klafter, "Lévy walks," Reviews of Modern Physics, vol. 87, no. 2, pp. 483-530, 2015.

[11] H. L. V. Trees, Detection, Estimation, and Modulation Theory, Part I. John Wiley and Sons, Inc, 2004.

[12] L. Chen, "On the convergence of Poisson binomial to Poisson distributions," The Annals of Probability, vol. 2, no. 1, pp. 178-180, 1974.

[13] L. Le Cam, "An approximation theorem for the Poisson binomial distribution," Pacific J. Math., vol. 10, pp. 1181-1197, 1960.

[14] L. P. Kaelbling et al., "Reinforcement learning: A survey," Journal of Artificial Intelligence Research, vol. 4, pp. 237-285, 1996.

[15] A. Dvoretzky et al., "Asymptotic minimax character of the sample distribution function and of the classical multinomial estimator," The Annals of Mathematical Statistics, vol. 27, no. 3, pp. 642-669, 1956. 